\section{Revista dePolítica Económica y Desarrollo Sostenible}

Centro Internacional de Política Económica para el Desarrollo Sostenible
Revista de Política Económica y Desarrollo Sostenible

EISSN: 2215-4167 • Vol. 1 (1) • Julio-Diciembre, 2015: 1-19

DOI: http://dx.doi.org/10.15359/peds.1-1.2

URL: http://www.revistas.una.ac.cr/politicaeconomica

Revista electrónica semestral publicada por el Centro Internacional de Política Económica para el Desarrollo Sostenible

Universidad Nacional, Lagunilla, Heredia Apartado 2393-3000

\title{
Impacto de la diversidad y la participación de los trabajadores sobre la innovación de las empresas: Un modelo de regresión en dos etapas para el sector servicios
}

\author{
Impact of Workforce Diversity and Participation on Enterprise Innovation: A \\ Two-Stage Regression Model for the Service Sector
}

Keynor Ruiz Mejías ${ }^{1}$, Rodrigo Corrales Mejías ${ }^{2}$

Fecha de recepción: 19.09.2014 Fecha de aceptación: 3.02.2015 Fecha de publicación: 17.11.2015

\begin{abstract}
Resumen
Tradicionalmente, los procesos de innovación han estado vinculados a las inversiones que se realizan en investigación y desarrollo (I+D), o bien, a las inversiones en capacitación del personal. Esto ha llevado a que los estudios sobre innovación se concentren en aspectos de avance tecnológico, dejando un poco de lado procesos incrementales de cambio y mejora que se traduzcan, eventualmente, en transformaciones sustanciales en las empresas. En el presente artículo se intenta determinar el efecto que tienen otros factores como la diversidad y la participación de los trabajadores sobre la innovación en las empresas. El factor diversidad aborda aspectos de composición según sexo y nivel educativo de la fuerza laboral de las empresas; mientras que el factor participación tiene que ver con los espacios de discusión de ideas, el origen de la iniciativa para la participación en procesos de cambio y el involucramiento en diferentes fases de procesos innovadores. Mediante un modelo de regresión Heckman, se busca determinar de manera bietápica las implicaciones de las variables en mención, sobre la innovación de las empresas del sector servicios. El primer apartado conceptualiza el término innovación. La segunda sección se centrada en explicar las vinculaciones teóricas de las variables seleccionadas sobre la innovación, junto con la estadística descriptiva que evidencia el comportamiento de las variables. Una tercera sección se dedica a la especificación del modelo y en la cuarta sección se presentan los principales resultados, cerrando con implicaciones de política que surgen de los resultados propios del modelo.
\end{abstract}

Palabras Clave: Diversidad por sexo, espacios de participación en las empresas, Innovación en servicios.

\begin{abstract}
Traditionally, innovation processes have been associated to investments in research and development (R\&D) or in staff training. This has led innovation studies to focus on technological advance issues leaving aside, sometimes, incremental processes of change and improvement that will eventually become substantial
\end{abstract}

1 Investigador y docente del Centro Internacional de Política Económica para el Desarrollo Sostenible (CINPE) de la Universidad Nacional. keynor.ruiz.mejias@una.cr

2 Investigador y docente del Centro Internacional de Política Económica para el Desarrollo Sostenible (CINPE) de la Universidad Nacional. rodrigo.corrales.mejias@una.cr 
Revista de Política Económica • EISSN: 2215-4167

Vol. 1 (1) • Julio-Diciembre, 2015: 1-19

DOI: http://dx.doi.org/10.15359/peds.1-1.2

Ruiz Mejías y Corrales Mejías

URL: http://www.revistas.una.ac.cr/politicaeconomica

changes in firms. In this work, we attempt to determine the impact of other factors such as workforce diversity and participation on enterprise innovation. The diversity factor relates to organizational composition aspects by workforce's sex and education level; the participation factor deals with spaces for discussion of ideas, origin of participation initiatives in processes of change, and involvement in the different phases of the innovation processes. A two-step Heckman regression model is used to determine the implications of the aforementioned variables on innovation of enterprises in the service sector. The first section of the paper conceptualizes innovation; the second section focuses on explaining the theoretical associations of selected variables on innovation together with descriptive statistics, which evidences the behavior of variables. A third section is devoted to the model's specification, and the fourth section shows the main results and, finally, a series of policy implications arising from the results of the model.

Keywords: diversity by sex, spaces for participation in firms, innovation on services.

\section{Introducción}

Los estudios sobre la dinámica de innovación se han centralizado en los procesos de avance tecnológico, los cambios y las mejoras radicales que por lo general responden a la implementación de equipo de alta tecnología (Nelson, 1993); dejando de lado otras variables clave que complementan el entendimiento de los procesos de innovación en las empresas. Algunas de ellas son incrementales, con repercusiones importantes tanto en los bienes, servicios y procesos productivos de las empresas, como en sus organizaciones y dinámicas de comercialización.

Por este motivo, el propósito principal de este artículo radica en analizar otros posibles factores que pueden estar influyendo en que las empresas realicen innovaciones, no solo para conocer su incidencia en procesos de cambio y mejora, sino además para poder inferir sobre las implicaciones de política en un espectro más amplio de variables que impulsan la innovación en las empresas. En ese sentido, se ha considerado la inclusión de aspectos de diversidad y los que se refieren a la participación activa de la fuerza laboral. Ambos son elementos identificados fundamentales para poder entender y explicar los procesos de innovación, remarcados por Lundvall $(1992,2002)$ y Edquist (1997), tanto al considerar el marco del análisis de los Sistemas Nacionales de Innovación, como los procesos basados en la creación de capacidades de aprendizaje que tienen lugar en el nivel de las personas y de las organizaciones.

Investigaciones como la de Zúñiga (2004) demuestran que la capacidad innovadora de las empresas depende en buena medida de las capacidades y competencias de su fuerza de trabajo, lo cual implica que la inversión en capacitación se considere como parte de la estrategia para el buen desempeño y la competitividad de las empresas, a través de la innovación. Por ello, la forma cómo se organice el trabajo, la interacción entre la administración y los trabajadores, y entre los mismos trabajadores, e inclusive algunas características demográficas de la composición laboral dentro de las empresas, han evidenciado ser factores que influyen de una u otra forma en la innovación (Ruíz, 2007; Østergaard et al., 2011; Corrales, 2013). 
Por esta razón, se decide utilizar en esta investigación las siguientes variables: el origen de la interacción en ideas innovadoras, la participación de los trabajadores en procesos de innovación, los espacios de discusión de ideas, la inversión en capacitación y en investigación y desarrollo (I+D). Junto con estas variables, se utilizaron las variables cognitivas y demográficas de nivel educativo de la fuerza laboral y la estructura laboral por sexo en las empresas. Los datos para la construcción de estas variables se tomaron de la Encuesta Nacional de Ciencia, Tecnología e Innovación del sector servicios costarricense para el año 2012 (MICITT, 2014), de la cual se dispuso de un total de 360 empresas (pequeñas, medianas y grandes) de subsectores financieros, salud, software y turismo.

El documento está estructurado en cinco secciones. En esta primera sección se ha introducido el tema; mientras que en la segunda sección se expone la justificación y fundamentación teórica de las variables elegidas y su vinculación con la innovación. Una tercera sección presenta las especificaciones y condiciones del abordaje metodológico utilizado. Una cuarta sección se encarga de presentar los principales resultados que surgen del modelo econométrico utilizado. Finalmente, una quinta sección concluye con las principales implicaciones de política económica con base en los resultados obtenidos.

\section{Fuerza de trabajo e innovación}

\subsection{Participación de los trabajadores en la generación de ideas innovadoras}

Los procesos de innovación tienden a reflejar las iniciativas y la creatividad de los trabajadores en su quehacer habitual. Lo anterior no como un hecho aislado, sino como parte de las características propias del proceso productivo, la organización del trabajo, la comunicación y la difusión del conocimiento, así como de los programas de capacitación, entre otros aspectos (Lundvall et al., 2001). Siguiendo la línea de Orozco (2002), la innovación entonces se concibe como un proceso interactivo entre diferentes actores que implica, a menudo, que exista cierto grado de comunicación e interacción entre los trabajadores dentro de las empresas. Asimismo, para Lundvall (1992), ello se da basado en las diferentes cualificaciones que puedan existir en diferentes niveles organizacionales (Lundvall, 1992).

En este sentido, la existencia de espacios para discusión de ideas, sugerencias y posibles cambios dentro de las empresas representa un mecanismo para potenciar el surgimiento de ideas innovadoras. Los espacios interactivos de aprendizaje (Arocena y Sutz, 2002), entendidos como situaciones que ofrecen oportunidades para aprender durante la búsqueda de soluciones a un problema dado, suelen representar los principales mecanismos utilizados por las empresas para obtener ideas de los trabajadores, dinámica que se realiza involucrando organizaciones, personas variadas y ámbitos diversos. Las reuniones formales o no formales, los buzones de sugerencias y los sistemas de 'puertas abiertas' para la recepción de nuevas ideas, representan mecanismos que 
Revista de Política Económica • EISSN: 2215-4167

Vol. 1 (1) • Julio-Diciembre, 2015: 1-19

DOI: http://dx.doi.org/10.15359/peds.1-1.2

Ruiz Mejías y Corrales Mejías

URL: http://www.revistas.una.ac.cr/politicaeconomica

incentivan la interacción entre la administración y los trabajadores y que, por tanto, se utilizan para extraer ideas innovadoras de quienes están en constante contacto con todo el proceso productivo de las empresas.

Por lo general, según Ruiz (2007), la estructura organizacional de la administración de las empresas tiende a ser mucho más vertical, mientras que en el área operativa la estructura tiende a ser mucho más horizontal. Esto, de acuerdo con Ruiz (2007:140), es una característica que sugiere que las empresas suelen tener una organización interna en forma de "L", donde el flujo de nuevas ideas y conocimiento ocurre en ambas direcciones: el vértice de esa forma en "L" constituye la representación de la interacción entre ambas áreas, la cual podría facilitar o dificultar, en gran medida, el flujo de conocimiento.

La evidencia muestra que precisamente los sistemas de 'puertas abiertas' para la recepción de nuevas ideas, en conjunto con reuniones formales con diferentes grupos de trabajo (conocidas también como brainstorming), son los mecanismos más utilizados por las empresas para involucrar a los trabajadores en el proceso creativo de generación de nuevas ideas. Estos sistemas representan un $83,1 \%$ y un 73,3 \% de los casos, respectivamente (MICITT, 2014). En efecto, el 96,2 \% de las empresas innovadoras afirman contar con espacios para la discusión de ideas (MICITT, 2014).

La innovación puede surgir de diferentes fuentes (Dijk y Sandee, 2002); en ese sentido, los dueños y administradores de las empresas se encuentran en la búsqueda constante de mejoras en lo que ofrecen y en cómo lo ofrecen, razón por la cual la atención a las ideas que provengan de los trabajadores según su experiencia, o sugerencias de fuentes externas como los clientes por ejemplo, resultan de gran importancia. A raíz de esto es que se considera, además, al origen de las ideas como una de las variables encargadas de explicar la innovación.

Existe evidencia en los sectores costarricenses de servicios (Corrales, 2013) y manufacturero (Ruiz, 2007), de que el origen de ideas innovadoras en las empresas yace en un esfuerzo conjunto entre los dueños, gerentes y trabajadores principalmente, siendo los dueños y gerentes los responsables más por innovaciones de producto (bien o servicio), mientras que los trabajadores se involucran más en innovaciones de proceso. Asimismo, según MICITT (2014), para el 2012 en el sector servicios costarricense, el 69,4\% de las empresas menciona que la mayor parte de la iniciativa para propiciar la participación de los trabajadores en la generación de ideas proviene de la gerencia, mientras que el 38,3 \% de los casos el origen de la participación proviene mayoritariamente de los mismos trabajadores.

De igual manera, otra de las variables de interés es la fase a partir de la cual se involucran los trabajadores en los procesos innovadores (fase de la idea, de decisión, de planeación o de ejecución). Se ha demostrado que el flujo de conocimiento por sí solo no produce innovación, sino que requiere de mecanismos que hagan la innovación más inclusiva (Corrales, 2014), por lo que la incorporación de los trabajadores en distintas fases del proceso productivo suele incentivar la innovación. Además, el conocimiento y la experiencia de los trabajadores, al ser quienes en 
Revista de Política Económica • EISSN: 2215-4167

Vol. 1 (1) • Julio-Diciembre, 2015: 1-19

DOI: http://dx.doi.org/10.15359/peds.1-1.2

Ruiz Mejías y Corrales Mejías

URL: http://www.revistas.una.ac.cr/politicaeconomica

muchos casos se encuentran en contacto más directo y constante con los procesos, productos y hasta con el cliente mismo, se convierten en actores clave para la generación de nuevas ideas dirigidas a cambios y mejoras (Knudsen, 1995; Wiig, 1997; Barney y Wright, 1998; Edquist et al., 2001; Hitt et al., 2001; Lam, 2002).

La innovación como un resultado no debe verse como un proceso aislado o incluso individualizado, ya que requiere de los esfuerzos coordinados de diferentes actores y de la integración de actividades a través de funciones especializadas, áreas de conocimiento y contextos de aplicación (Lam, 2010), donde las iniciativas innovadoras no estén a cargo de una persona, sino de la interacción social y de la difusión e integración del conocimiento en las empresas. Asimismo, la innovación es un proceso de aprendizaje y el aprendizaje es un proceso colectivo que ocurre dentro de un escenario organizado, esto convierte a la innovación en un proceso de aprendizaje organizacional y creación de conocimiento (Lam, 2010). En ese sentido, si la innovación es conocimiento, la manera en cómo este fluya dentro de las empresas depende de la forma en que estas se organizan, de forma tal que los espacios de participación dentro de la organización deban entenderse como un estímulo a la innovación.

En el Informe Nacional de Ciencia, Tecnología e Innovación 2012, se detalla que en el sector servicios costarricense el $41,9 \%$ de los trabajadores tienden a involucrarse en la fase de la idea en lo que refiere a cambios y mejoras en las empresas. Esta mayor participación se concentra en ideas generadoras de nuevos productos o procesos. Las empresas mencionan que un 36,7 \% de los trabajadores se involucran a partir de la fase de ejecución y solamente en un 2,5\% de los casos afirman que los trabajadores se involucraron en la fase de decisión. Estos datos refuerzan, en cierta medida, la relevancia del rol que juegan los trabajadores en la generación de nuevas ideas dentro de las empresas, mientras que la toma de decisiones sobre la implementación o no de innovaciones sigue estando en manos de quienes manejen los negocios.

\subsection{Diversidad en las empresas: Dimensiones demográficas y cognitivas de los trabajadores}

Para medir el grado de diversidad entre los trabajadores es necesario utilizar características que tiendan a influir sobre su experiencia y base de conocimiento, la educación formal y la identidad cultural, por lo que estas pueden ser clasificadas en características que forman parte de cada individuo (atributos demográficos como el sexo, etnicidad o nacionalidad) y características logradas como lo son el nivel educativo o la experiencia laboral (Ruet et al., 2003). Precisamente, la variable sexo, entendida como la composición porcentual de hombres y mujeres que conforman la estructura laboral en las empresas, y el nivel de educación de los trabajadores, representan variables clave que ya han sido evidenciadas en diversos estudios como determinantes de la innovación (Bantel y Jackson, 1989; Lazear, 1999; Østergaard et al., 2011; Parotta et al., 2014).

La variedad en los niveles de formación, experiencia y habilidades entre los trabajadores tienden a propiciar complementariedades entre los trabajadores que fomentan el desarrollo de 
Revista de Política Económica • EISSN: 2215-4167

Vol. 1 (1) • Julio-Diciembre, 2015: 1-19

DOI: http://dx.doi.org/10.15359/peds.1-1.2

Ruiz Mejías y Corrales Mejías

URL: http://www.revistas.una.ac.cr/politicaeconomica

competencias en otras áreas más allá de las que inicialmente corresponde a cada trabajador (Dosi, 1988; Quintana-Garca y Benavides-Velasco, 2008). Un ejemplo de las características propias de estructuras laborales diversas hace referencia a la presencia de trabajadores de ambos sexos o trabajadores con distintos niveles de instrucción (profesionales, técnicos, entre otros).

Algunos factores, como la formación vocacional y la experiencia, dentro de la dimensión cognitiva, y la edad, el trasfondo cultural y el sexo, dentro de la dimensión demográfica, intervienen en la aplicación y combinación del conocimiento existente, así como en la comunicación e interacción entre los trabajadores. De esta forma, la diversidad de características de los trabajadores tendría un efecto positivo en la innovación al crear espacios de discusióninteracción mucho más amplios, que permiten a las empresas estar mucho más propensas a la generación de nuevas ideas e iniciativas creativas (Østergaard et al., 2011), en tanto esa diversidad contribuya a la creación de nuevas combinaciones de conocimiento (Schumpeter, 1934).

La diversidad resulta un factor importante para entender la innovación, en tanto la variedad existente pueda contribuir a la complementariedad del conocimiento de base (van der Vegt y Jenssen, 2003; Østergaard et al., 2011). A pesar de que los estudios que vinculan la innovación con la composición según sexo de la fuerza laboral son escasos, la evidencia existente señala un efecto positivo hacia la innovación en aquellas firmas cuya estructura laboral se encuentra relativamente balanceada (relación 50-50 o 60-40 entre hombres y mujeres), mientras que en casos donde hubiese mayor concentración de un único sexo, las empresas tienden a ser menos innovadoras (Østergaard et al., 2011; Andries et al., 2014). Es por ello que los aspectos alusivos a la variable sexo no deben ser ignorados dentro de los estudios sobre innovación que focalizan la diversidad de la estructura laboral.

Según MICITT (2014), en el sector servicios costarricense, solo alrededor del $5 \%$ de las empresas que indicaron haber realizado algún tipo de innovación poseen una estructura laboral con una predominancia muy pronunciada de un único sexo (más del $90 \%$ de los trabajadores son del mismo sexo), mientras que el $56,3 \%$ de las empresas innovadoras indicaron tener una fuerza laboral relativamente balanceada entre hombres y mujeres (entre el 50 y el $70 \%$ de los trabajadores del mismo sexo).

En lo que respecta al nivel de educación de los trabajadores, se ha demostrado que la diversidad en las distintas cualificaciones formales de la fuerza laboral tiende a crear un ambiente de intercambio de conocimiento y aprendizaje que propicia los procesos de innovación, constituyendo además una parte importante de la capacidad de absorción de las empresas ${ }^{3}$ (Cohen y Levinthal, 1990; Henderson y Clark 1990; Kogut y Zander 1992; Nonaka 1991; Nonaka y Takeuchi 1995; Drazin y Rao 2002; Lundvall, 2002). Estudios como el de Østergaard et al. (2011) y Parrotta et al. (2014) muestran que las empresas con cantidades mayores de personal con niveles altos de educación, tienden a ser más innovadoras.

3 El término anglosajón absorptive capacity de las empresas hace referencia a la "habilidad de la empresa para identificar, asimilar y explotar el conocimiento del ambiente" (Cohen y Levinthal, 1990: 569). 
Revista de Política Económica • EISSN: 2215-4167

Vol. 1 (1) • Julio-Diciembre, 2015: 1-19

DOI: http://dx.doi.org/10.15359/peds.1-1.2

Ruiz Mejías y Corrales Mejías

URL: http://www.revistas.una.ac.cr/politicaeconomica

Para el caso costarricense, en el sector servicios propiamente se tiene que el $68 \%$ de las empresas que realizaron innovaciones en el 2012 cuentan con trabajadores profesionales en áreas tales como ciencias exactas, naturales, médicas, agrícolas, gastronómicas y sociales, así como en ingenierías, humanidades y profesionales en turismo (MICITT, 2014).

\subsection{Variables básicas para explicar la innovación}

En el análisis se incorporan dos variables que son determinantes para la innovación. La primera de ellas es la inversión que realizan las empresas en investigación y desarrollo (I+D). La inversión en I+D, si bien es un determinante que se suele vincular principalmente a las innovaciones tecnológicas (Freeman, 1987), su no inclusión dentro del análisis podría llevar a errores de especificación. Asimismo, la I+D es responsable también por propiciar innovaciones incrementales (Lundvall, 2007), en tanto estas acciones no se limiten únicamente a laboratorios o invenciones científicas, sino que además pueden corresponder a investigaciones menos pretenciosas que igualmente puedan generar cambios, mejoras e ideas novedosas para las empresas. Para el caso de los servicios en Costa Rica, en el 2012 el 41 \% de las empresas que realizaron innovaciones llevaron a cabo inversiones en I+D interna, externa o ambas (MICITT, 2014).

La segunda variable es la inversión realizada en capacitación. En lo que respecta esta, Lundvall (2007) enfatiza que la acción de crear capacidades y competencias en los trabajadores a lo interno de las empresas constituye un primer paso hacia la generación de innovaciones. Es importante prever que la creación de capacidades no depende exclusivamente de lo que los trabajadores aprenden antes de incorporarse a la fuerza de trabajo, el tener trabajadores a la medida es una responsabilidad de cada empresa, a través de mecanismos como la educación dual o maestro-aprendiz, u otras formas de creación de competencias. De esta forma se refiere también a la atmósfera en la cual se desarrollan los individuos y cómo esta promueve el potencial de aprendizaje (Ruiz, 2007).

Se evidencia la importancia de la capacitación en el sector empresarial, pues en el 2012 el $80,4 \%$ de las empresas de servicios que realizaron innovaciones mencionaron haber invertido en capacitación como parte de sus actividades dirigidas a generar procesos de innovación.

\section{Un modelo Heckman para explicar la innovación}

Para esta investigación, se utilizan los datos de la Encuesta Nacional de Ciencia, Tecnología e Innovación 2012, la cual corresponde a información del sector servicios costarricense. Sin embargo, la información de las observaciones corresponde solamente a cuatro subsectores del sector servicios (turismo, sector financiero, salud y software), por lo que explicar la innovación utilizando una muestra que no represente la totalidad del sector genera un sesgo de selección a la hora de usar un modelo de regresión por mínimos cuadrados ordinarios como herramienta de análisis. Por este motivo, se utiliza un modelo Heckman (1979), en tanto este coadyuva a aislar 
Revista de Política Económica • EISSN: 2215-4167

Vol. 1 (1) • Julio-Diciembre, 2015: 1-19

DOI: http://dx.doi.org/10.15359/peds.1-1.2

Ruiz Mejías y Corrales Mejías

URL: http://www.revistas.una.ac.cr/politicaeconomica

los factores que afectan el proceso de selección de la muestra y reducir el sesgo de selección de los determinantes de la variable dependiente (Wooldridge, 2006).

\subsection{Variable dependiente}

La variable dependiente seleccionada para el estudio está constituida por las empresas que dijeron haber realizado alguna innovación (INN), la cual se mide como una variable categórica que indica si las empresas realizaron o no innovaciones durante el año en estudio. Para este caso, la innovación es entendida como la modificación o surgimiento de nuevos productos (bienes y servicios), procesos y formas organizacionales o de comercialización. La variable INN es explicada por un vector $z$ acompañado de lo los coeficientes a estimar y el término de error estocástico. El vector $z$ contiene las variables explicativas mencionadas a lo largo del documento. Por lo tanto, la variable dicotómica de INN tiene la siguiente forma:

Los modelos con variable dependiente de respuesta cualitativa pueden ser explicados con modelos Logit, Probit o Tobit, pero para este caso particular no resultan apropiados, ya que la variable dependiente se encuentra limitada o censurada, en tanto que solo es observada plenamente en una parte de su rango o de su población, como sucede en el caso de los datos del sector servicios, ocasionando así un sesgo de selección. Es por esto que al utilizar un modelo Heckman se puede descomponer el modelo en dos procesos que generen un modelo bivariante con dos ecuaciones estimadas básicamente por el método de Mínimos Cuadrados en dos etapas (González, 2010).

La especificación del modelo se hace por medio de dos ecuaciones: una ecuación de interés o principal, que corresponde a la ecuación que se busca estimar para la extracción de conclusiones y la explicación de la variable dependiente, y una ecuación de selección (regresión auxiliar), que corresponde a un modelo de elección discreta (Probit o Logit) que mide la probabilidad de estar en la muestra (Wooldrigde, 2006).

\subsection{Variables explicativas}

La regresión de interés incluye las variables explicativas de la innovación en las empresas, tales como la inversión en investigación y desarrollo (IDT) e inversión en capacitación (Capac), aunadas a las variables de corte demográfico (DivSex) y cognitivo (Educ).

La variable sexo expresa la diversidad en la composición según sexo de la estructura laboral de las empresas. Esta variable se subdividió en cinco grupos ${ }^{4}$, pues resultaría útil para el estudio el

4 El primer grupo (Sex1) correspondería a una composición de trabajadores entre 90-100 \% de personas del mismo sexo; el segundo grupo (Sex2) una composición entre 80-90 \% del mismo sexo; el tercer grupo (Sex3) una composición entre 70-80 \% del mismo sexo; un cuarto y quinto grupo (Sex4 y Sex5) con composiciones entre 60-70 \% y 50-60 \% del mismo sexo, respectivamente. Esto siguiendo la metodología utilizada por Østergaard et al., 2011. Asimismo, se procedió a realizar pruebas de diferencias de medias, lo que arrojó que las variables creadas no eran significativamente distintas, lo que aunó a la decisión de excluir este método. 
Revista de Política Económica • EISSN: 2215-4167

Vol. 1 (1) • Julio-Diciembre, 2015: 1-19

DOI: http://dx.doi.org/10.15359/peds.1-1.2

Ruiz Mejías y Corrales Mejías

URL: http://www.revistas.una.ac.cr/politicaeconomica

poder determinar si una composición más -o menos- balanceada de trabajadores surte algún efecto sobre la innovación. Sin embargo, el análisis descriptivo de los datos entre empresas innovadoras y no innovadoras no mostró evidencia de que los subgrupos pudiesen explicar la innovación; mientras que al tratarla como una sola variable (DivSex) que midiera la presencia o no de diversidad, tomando como criterio la prevalencia de composiciones laborales de entre 50 y $70 \%$ de trabajadores del mismo sexo, se encuentran patrones que podrían aportar significancia al análisis.

Por otra parte, la variable nivel de educación $(E d u c)$ corresponde a la presencia de profesionales dentro de las empresas (incluyendo entre otros profesionales de ingenierías u otras ciencias duras). Como se explica anteriormente, esta variable se incluye dada la evidencia de que la presencia de profesionales en las empresas contribuye positivamente a la innovación (Østergaard et al., 2011; Parrotta, 2014). Se elige trabajar con la categoría 'profesionales', como variable proxy de nivel alto de educación ya que se ajusta a los datos que se poseen de la Encuesta Nacional.

En lo que respecta a la ecuación de selección, se incluyen las variables explicativas que aportan una mayor comprensión de la relación entre participación de los trabajadores y la innovación en las empresas.

La primera variable explicativa es la presencia o no de espacios para la obtención y discusión de ideas entre los patronos y los trabajadores dentro de las empresas (Espac). La variable contempla aspectos como la existencia de buzones para depositar ideas, reuniones formales y no formales (brainstorming), reuniones individuales o sistemas de puertas abiertas.

También, se incorpora la variable que indica si en las empresas, la participación de los trabajadores en la gestión de procesos de innovación (Particip). Esta variable se subdivide en caso de que la participación sea en la fase de generación de ideas (Particip1), de decisión (Particip2), de planeación (Particip3) o de ejecución (Particip4). Aunado a esto, se utiliza como variable independiente al origen de la iniciativa de participación en procesos de cambio y mejora (Origen), el cual puede hacer referencia a los trabajadores (Origen1), a la gerencia (Origen2), a los dueños (Origen3) o a alguna influencia externa (Origen4).

Estas dos últimas variables se incluyen dentro del modelo en subagrupaciones, es decir, se incluye una variable dicotómica por cada opción de respuesta, esto para poder determinar el efecto de cada opción en la probabilidad de obtener o no innovaciones. En el caso de Particip, se incorporan cuatro variables dicotómicas para cada fase en la que se involucran los trabajadores, mientras que para la variable Origen, se incluyen cuatro variables dicotómicas para referirse al agente del cual proviene la iniciativa de participación. La variable Particip, al haber sido en la encuesta una variable de respuesta única (cada opción a elegir es mutuamente excluyente de las otras), puede ocasionar que el modelo se vea afectado por la trampa de la variable dicotómica (Gujarati, 2010), por lo que se aclara que en esta ecuación de selección ${ }^{5}$ se omitirá la constante en aras de evitar problemas de multicolinealidad.

5 Lo mismo ocurre en la ecuación de interés al conformarse de variables binarias con las mismas condiciones. 
Revista de Política Económica • EISSN: 2215-4167

Vol. 1 (1) • Julio-Diciembre, 2015: 1-19

DOI: http://dx.doi.org/10.15359/peds.1-1.2

Ruiz Mejías y Corrales Mejías

URL: http://www.revistas.una.ac.cr/politicaeconomica

\subsection{Especificación del modelo Heckman}

Las características de las variables elegidas pueden llevar a la conclusión precipitada de utilizar un modelo de regresión lineal por el método de mínimos cuadrados ordinarios (MCO), o bien, por tratarse de una variable dependiente de respuesta binaria, utilizar entonces un modelo Probit o Logit. No obstante, desde el inicio de la investigación y por las características de la muestra, se sospecha la presencia de un sesgo de selección, razón por la cual si se estima un modelo mediante el método de MCO, existiría la posibilidad de obtener unos estimadores sesgados, lo que llevaría a conclusiones erróneas. Para comprobar la presencia contundente de este problema, resultó necesario realizar primero una prueba con la inversa del ratio de Mills, que comúnmente se representa con la variable Lambda $(\lambda)$. Este parámetro se utiliza para probar la hipótesis ${ }^{6}$ que indica la presencia o no de sesgo de selección, el cual al realizar el test de significancia mediante la prueba del valor p, se concluye que es significativo al $95 \%$ de confianza. Esto en efecto indica que la estimación del modelo por MCO estaría sesgado, lo que demanda la utilización de un modelo en dos etapas que permita corregir el problema de sesgo de selección.

Un modelo Heckman en dos etapas estima el modelo por mínimos cuadrados en dos etapas. En la primera etapa se estima, mediante el método de máxima verosimilitud, un modelo Logit o Probit que permita determinar la probabilidad de que las variables incluidas en la ecuación de selección (auxiliar) influyan sobre la variable dependiente. Posteriormente, en la segunda etapa se estima la ecuación de interés donde se incorporan, ahora sí, las variables explicativas principales del modelo. Una vez realizadas ambas regresiones, se debe evaluar nuevamente el parámetro lambda, que de resultar significativo en esta nueva regresión, se puede afirmar que se ha corregido el sesgo de selección mediante el modelo Heckman (Wooldridge, 2006). En efecto, para el caso en estudio, el valor de lambda resultó de 0,003 por lo que se puede concluir que mediante el Heckman en dos etapas se corrige el sesgo de selección y se puede trabajar con los mejores estimadores lineales insesgados (MELI) ${ }^{7}$.

Las ecuaciones de interés y selección, respectivamente, para las variables definidas son las siguientes $^{8}$ :

La variable dependiente hace referencia a la probabilidad de que las variables explicativas de la ecuación de selección tengan algún efecto sobre la innovación como variable dependiente en la ecuación de interés; mientras que denota la función de densidad normal estándar.

6 Para este caso se trabaja con las siguientes hipótesis ; indicando que si el ratio de Mills es igual a cero, no existe un sesgo de selección. El resultado de la modelística arroja que el valor de lambda es de 0,000 por lo que se rechaza la hipótesis nula a un nivel de significancia del $1 \%$, concluyendo la presencia de un sesgo de selección.

7 Es importante aclarar que no se utilizó en la regresión la opción de errores estándar robustos, debido a que no es un método compatible en el procedimiento bietápico y por el riesgo de que pueda introducir errores en la regresión (Freeman, 2006).

8 Se recuerda que las variables Particip y Origen se subdivide cada una en las categorías explicadas, sin embargo por razones de simplicidad en las ecuaciones se presenta solo la variable explicativa en forma general, denotando además que el signo esperado para todas las variables es positivo por la relación directa explicada previamente en este documento. 
Revista de Política Económica • EISSN: 2215-4167

Vol. 1 (1) • Julio-Diciembre, 2015: 1-19

DOI: http://dx.doi.org/10.15359/peds.1-1.2

Ruiz Mejías y Corrales Mejías

URL: http://www.revistas.una.ac.cr/politicaeconomica

\section{Resultados}

Para determinar la relación de las variables explicativas con la innovación, se realizan dos regresiones con variaciones en el modelo. El primer modelo, tal y como se presenta en el cuadro 1, consiste en un modelo de regresión bietápico donde se incluyen todas las variables seleccionadas para el estudio. En esta primera regresión algunas variables no resultaron significativas, indicando su poca o nula relación estadística con la innovación. La significancia resultante de las variables explicativas se puede complementar con lo que muestra la estadística descriptiva, pues variables como la participación de los trabajadores en la fase de decisión (Particip2) y la influencia externa sobre el origen de la iniciativa para la cooperación trabajador-patrono (Origen4), no fueron determinantes para las empresas que realizaron alguna innovación.

A partir de esos resultados, se ejecuta un segundo modelo donde se excluyen las variables que no resultaron significativas y que, según la evidencia, mostraban poca relación con la innovación en las empresas. Los resultados del modelo 2 señalan que en su gran mayoría, todas las variables incluidas muestran una relación positiva significativa con las empresas que realizaron algún tipo de innovación. Además de la significancia demostrada de las variables básicas como IDT y CAPAC, se recalca la importancia de incluir variables de diversidad (DivSex y Educ) y de participación (Particip, Origen y Espac), para poder explicar la innovación en las empresas, en tanto la evidencia muestra tener influencia sobre los procesos de innovación.

Los resultados muestran una relación importante entre la inversión en capacitación y en investigación y desarrollo y las empresas innovadoras. En este sentido, la capacitación no solo es la actividad de innovación más utilizada por las empresas, sino que en efecto evidencia su impacto sobre procesos innovadores en las mismas. Por otra parte, en cuanto a I+D, aun cuando solo el 40 \% de las empresas innovadoras realizaron este tipo inversión, se evidencia una estrecha relación entre este aspecto y la incidencia en innovación, lo que se explica principalmente por el peso relativo que la $\mathrm{I}+\mathrm{D}$ adiciona a empresas que realizaron innovaciones radicales.

Las variables de sexo y nivel de educación, tal y como han sido abordadas por diversos autores (Østergaard et al., 2011; Andries et al., 2014; Parrotta et al., 2014), demostraron tener un peso importante sobre las empresas innovadoras. Esto permite inferir que en el sector servicios aspectos como una estructura laboral balanceada entre hombres y mujeres, así como la presencia de trabajadores con un nivel educativo alto, influyen sobre los procesos de innovación en las empresas.

Con respecto a las variables de participación, se demuestra que el involucramiento de los trabajadores en la fase de planeación ${ }^{9}$ de cambios y mejoras tiene un efecto positivo sobre la innovación. De esta forma, se puede afirmar que la participación de los trabajadores puede representar una de las principales fuentes para crear conocimiento nuevo, sobre todo a través de su experiencia con el quehacer diario y habilidades propias (Grant, 1997), lo que a su vez se complementa con el interés por parte de las empresas en invertir en la capacitación de sus trabajadores.

9 Variables como participación en la fase de las ideas (Particip1) o en la fase de ejecución (Particip4), aunque teóricamente tienen una relación estrecha con la innovación en las empresas, no resultaron significativas. La razón instrumental se basa en que los porcentajes de participación en estas fases, tanto para las empresas innovadoras como para las que no realizaron innovaciones en el año de referencia, fueron similares, por lo que se presenta la situación de no poder separar el efecto participación de estas variables sobre la variable dependiente. 
Revista de Política Económica • EISSN: 2215-4167

Vol. 1 (1) • Julio-Diciembre, 2015: 1-19

DOI: http://dx.doi.org/10.15359/peds.1-1.2

Ruiz Mejías y Corrales Mejías

URL: http://www.revistas.una.ac.cr/politicaeconomica

Asimismo, se encuentra que existe una relación positiva entre el interés que tienen tanto los trabajadores del área operativa, como los gerentes de las empresas para vincularse en los procesos de cambio y mejora. Esto, aunado a la participación de los trabajadores en la generación de ideas, indica de manera general que las empresas innovadoras poseen estructuras organizacionales que tienden a la forma en "L" que explica Ruiz (2007), donde las vinculaciones entre la administración y los trabajadores del área operativa permiten un flujo de conocimiento tal cual, eventualmente, genere procesos de innovación. El vértice de esta forma organizacional en "L" se explica por la existencia de espacios de participación para discutir nuevas ideas así como propuestas de cambio y mejora y que, en efecto, según los resultados del modelo, tienen un impacto positivo sobre las empresas innovadoras.

Con esto, se recalca la importancia de comenzar a incorporar, dentro del análisis de la innovación, variables que puedan medir la participación de los trabajadores en los diferentes procesos que conlleven a innovar, pues permiten de cierta forma entender los procesos de innovación de una manera más integral.

Cuadro No 1. Resumen del análisis de regresión

\begin{tabular}{|c|c|c|c|c|c|c|c|c|}
\hline \multicolumn{3}{|c|}{ Variables } & \multicolumn{3}{|c|}{ Modelo 1} & \multicolumn{3}{|c|}{ Modelo 2} \\
\hline INN Variable Dependiente & Coef. & S.E. & $\mathrm{z}$ & $\mathrm{P}>|\mathrm{z}|$ & Coef. & S.E. & $\mathrm{z}$ & $\mathrm{P}>|\mathrm{z}|$ \\
\hline DivSex & 0,136 & 0,036 & 3,81 & $0,000^{* * *}$ & 0,128 & 0,033 & 3,90 & $0,000^{* * *}$ \\
\hline Educ & 0,172 & 0,035 & 4,86 & $0,000^{* * *}$ & 0,176 & 0,037 & 4,81 & $0,000^{* * *}$ \\
\hline IDT & 0,100 & 0,040 & 2,49 & $0,013^{\star *}$ & 0,109 & 0,038 & 2,87 & $0,004^{* * *}$ \\
\hline Capac & 0,411 & 0,048 & 8,65 & $0,000^{* * *}$ & 0,422 & 0,043 & 9,83 & $0,000^{* * *}$ \\
\hline Ecuación de Selección & & & & $0,075^{\star}$ & & & & 0,338 \\
\hline Particip1 & 0,639 & 0,359 & 1,78 & & 0,177 & 0,184 & 0,96 & \\
\hline Particip2 & 0,597 & 0,665 & 0,90 & 0,369 & & & & \\
\hline Particip3 & 0,894 & 0,397 & 2,25 & $0,024^{\star *}$ & 0,457 & 0,269 & 1,70 & 0,090 * \\
\hline Particip4 & 0,467 & 0,344 & 1,36 & 0,175 & & & & \\
\hline Origen 1 & 0,336 & 0,189 & 1,77 & $0,076^{\star}$ & 0,325 & 0,186 & 1,75 & $0,080^{*}$ \\
\hline Origen 2 & 0,352 & 0,187 & 1,88 & 0,060 * & 0,343 & 0,178 & 1,93 & $0,054^{*}$ \\
\hline Origen 3 & $-0,253$ & 0,182 & $-1,39$ & 0,164 & & & & \\
\hline Origen 4 & $-0,078$ & 0,180 & $-0,43$ & 0,668 & & & & \\
\hline Espac & 0,443 & 0,333 & 1,33 & 0,184 & 0,729 & 0,160 & 4,56 & $0,000^{* * *}$ \\
\hline (Mills) Lambda & 1,564 & 0,172 & 9,09 & 0,000 & 1,460 & 0,161 & 9,09 & $0,000^{* * *}$ \\
\hline $\mathrm{N}$ & & 360 & & & 360 & & & \\
\hline Wald $\mathrm{Chi}^{2}(5)$ & & 126,19 & & & 214,11 & & & \\
\hline Prob $>\mathrm{Chi}^{2}$ & & 0,000 & & & 0,000 & & & \\
\hline rho & & 1,723 & & & 1,694 & & & \\
\hline sigma & & 0.907 & & & 0,862 & & & \\
\hline $\begin{array}{l}* \text { Significante al nivel de } 10 \\
\star * \text { Significante al nivel de } 5 \\
\star * \star \text { Significante al nivel de } 1\end{array}$ & & & & & & & & \\
\hline
\end{tabular}


Revista de Política Económica • EISSN: 2215-4167

Vol. 1 (1) • Julio-Diciembre, 2015: 1-19

DOI: http://dx.doi.org/10.15359/peds.1-1.2

Ruiz Mejías y Corrales Mejías

URL: http://www.revistas.una.ac.cr/politicaeconomica

\section{Implicaciones de política: Impulsos para promover la innovación}

La inversión que realizan las empresas tanto en $\mathrm{I}+\mathrm{D}$ como en capacitación son sin lugar a dudas dos variables claves en la generación de innovaciones. No obstante, en países en desarrollo son difíciles de estimular, porque ambas involucran recursos que ya se saben que son escasos. El esfuerzo propio de las empresas en general representa el 31,3 \% del total de inversión en I+D del país, el restante porcentaje está concentrado en centros públicos de investigación (universidades y otras entidades públicas). Por su parte, las empresas pequeñas y medianas tienen una inversión relativamente baja en términos absolutos (US\$7.603 y US\$17.123, respectivamente), pero con respecto a las ventas es significativamente importante (1,28 \% y $1,52 \%$, respectivamente), incluso más que el de las empresas grandes (0,83\%).

Esto significa que propiciar un aumento en la inversión en $\mathrm{I}+\mathrm{D}$ en estos grupos de empresas implica el poner a disposición grandes fondos que por sus características son de alto riesgo. Esta razón incide en que este tipo de inversiones no sean compatibles con el sistema de financiamiento tradicional. Por tanto, la evolución propia de un sistema de innovación más integrado sería no solo a partir del desarrollo de fondos de financiamiento individual (v.g. capital de riesgo), sino también la promoción de fondos que generen nuevos conocimientos y capacidades en el nivel sectorial o de grupo de empresas. En lo que se desea insistir es que ningún fondo alcanza para el financiamiento de totas las iniciativas individuales, claro que son importantes, pero no son la única opción y menos si son fondos no reembolsables. La vinculación Universidad-Empresa, las exoneraciones parciales del impuesto sobre la renta como porcentaje de la inversión en I+D, la reinversión de utilidades y la inversión de capital fresco mediante inversionistas privados, pueden ser opciones sistémicas que acompañen la dinámica empresarial para la generación de procesos de innovación. La distribución de la riqueza generada es un tema subsecuente cuando la estrategia es sistémica, no obstante ese tema trasciende los alcances de este documento.

En cuanto a los espacios de participación, si bien es cierto este ámbito radica en el nivel propio de las decisiones que se toman dentro de cada empresa, resulta un aspecto clave en materia de política para impulsar la innovación. La mayoría de empresas innovadoras afirmaron contar con espacios para la discusión de ideas, existiendo a su vez un grado importante de anuencia por parte de los gerentes y de los trabajadores a involucrarse en iniciativas de cooperación para la generación de nuevas ideas sobre cambios y mejoras en la producción. Con esto, se puede aprovechar el contar con formas de organización que faciliten el flujo de conocimiento entre las diferentes áreas (administrativa y operativa), en tanto propicien procesos innovadores. Muchas han sido las experiencias empresariales para procurar una mejor comunicación y una mayor participación de las personas trabajadoras de una empresa, algunas de ellas con resultados frustrantes para la misma empresa como un todo, quizá porque no estaban preparadas para estimular la participación y las personas encargadas de gestionarla no pudieron hacerlo adecuadamente. En otros muchos casos, la creación de espacios de participación ha brindado muy buenos resultados, claro está que no puede ser tomada a la ligera, debe haber administradores 
Revista de Política Económica • EISSN: 2215-4167

Vol. 1 (1) • Julio-Diciembre, 2015: 1-19

DOI: http://dx.doi.org/10.15359/peds.1-1.2

Ruiz Mejías y Corrales Mejías

URL: http://www.revistas.una.ac.cr/politicaeconomica

responsables para que las ideas no se diluyan y las reglas deben estar claras de cuál es el alcance de la propiedad intelectual de generar nuevas ideas para la empresa.

En ese sentido, no se debe olvidar que la innovación es conocimiento, por tanto, la forma en cómo se gestione el conocimiento y las ideas dentro de una organización (empresa), es determinante para estimular u obstaculizar dinámicas innovadoras. De igual forma, uno de los aspectos que se debe tener en consideración al tratar de propiciar espacios colaborativos de mayor compromiso por parte de los trabajadores hace referencia a la necesidad de cumplir como mínimo la institucionalidad laboral que establece un piso para potenciar las capacidades y competencias de los trabajadores en el nivel nacional y por ende en el nivel de empresa.

En cuanto a la diversidad según la composición por sexo de la fuerza laboral y el nivel educativo, pueden traducirse incluso como posibles estrategias de las empresas para propiciar la innovación. Los resultados muestran que las empresas innovadoras tienden a tener composiciones laborales relativamente balanceadas entre trabajadores de ambos sexos y, además, poseen un porcentaje importante de trabajadores profesionales. Lo anterior, para el caso analizado en el presente estudio, al igual que en Woodman et al. (1993) y Wenger (2000), propicia que a lo interno de las empresas existan diferentes combinaciones de conocimiento, habilidades y experiencias, y que a través de la interacción y el aprendizaje se genere nuevo conocimiento y, posteriormente, innovaciones. Claro está que no puede generalizarse sobre cuál es la composición adecuada en términos de sexo de las personas trabajadoras y su nivel de instrucción, y mucho menos sería prudente decirle a quienes conocen las necesidades de contratación de las empresas que esta variable es imperativa; sino que, si se pretende incluir un par de categorías de análisis a la hora de realizar contrataciones, una de ellas es la composición de los grupos de trabajo según sexo y la otra es la consideración es específico del nivel educativo, sus características y expresiones para el trabajo en equipo y el compromiso.

En el nivel del país, la decisión de continuar invirtiendo en la creación de capacidades y competencias de las personas tanto en el nivel técnico como en el nivel profesional (en igualdad de condiciones que propicie una participación para personas de ambos sexos en diferentes procesos productivos y áreas profesionales), se torna una estrategia estructural para impulsar una base común para dinamizar procesos de innovación en las empresas. Este es un tema ampliamente conocido en el país, en particular el rol de las organizaciones de formación profesional; sin embargo, no se deseaba dejar pasar por alto la importancia de recalcarlo, porque es clave continuar pensando en la regionalización, la renovación de carreras y cursos, la necesidad de trabajar estrechamente con quienes requieren personas capacitadas (nuevamente, no en el nivel individual, sino en el nivel sectorial), no solo para satisfacer la demanda actual, sino pensando en el futuro, en la importancia de formar no solo para ser empleados, sino también para desarrollar sus propias empresas, entre otros aspectos.

Relacionado con lo anterior, es importante tener en consideración que algunas veces es difícil contar con los recursos para tener permanentemente profesionales dentro de la empresa, 
por lo que resulta válido pensar en la posibilidad de fortalecer una iniciativa que estimule la interacción de profesionales con las empresas, sin que necesariamente sean trabajadores de la organización pero con el compromiso claro de lograr una innovación en la empresa o empresas (según sea la circunstancia). Una vinculación temprana con universidades que facilite la creación de proyectos conjuntos, convenios para prácticas profesionales supervisadas, programas de capacitación y acompañamiento y financiamiento con fondos para promover la innovación, entre otros mecanismos, no solo permite inducir a dotar a las empresas de personal más calificado, sino que además impulsa la constitución de un sistema nacional de innovación más sólido, porque se cimenta más en la interacción y vinculación que en el aislamiento.

Si bien los subsectores software, salud y financiero son dependientes de la presencia de profesionales en sus empresas, esta no necesariamente es la situación del subsector turismo; por tanto, los esfuerzos en servicios debe responder no solo a la oferta disponible, sino también a la demanda real de personal requerido para labores determinadas. Pero, además, debería existir una constante preocupación en la creación de capacidades y competencias nuevas en estos trabajadores, tanto por parte de las empresas, como por parte del gobierno, para definir como constante la evolución de los procesos productivos, según las condiciones mismas que el sistema económico demande, a través de la innovación.

\section{Referencias bibliográficas}

Andries, P., Czarnitzki, D. (2014). Small firm innovation performance and employee involment. New York: Springer Science Business Media (43) 21-38. Retrieved from: https://link. springer.com/content/pdf/10.1007\%2Fs11187-014-9577-1.pdf

Arocena, R. y Sutz, J. (2002). Sistemas de Innovación y Países en Desarrollo. Universidad de la República de Uruguay. SUDESCA. Recuperado de: http://portales.puj.edu.co/jaguilar/Paisesdesarrollo.pdf

Bantel, K., \& Jackson, S. (1989). Top management and innovation in banking: does the composition of the top team make a difference? Strategic Management Journal, 10, 107-124. Doi: https://doi.org/10.1002/smj.4250100709

Barney, J. B., \& Wright, P. M. (1998). On becoming a strategic partner: The role of human resources in gaining competi- tive advantage. Human Resource Management, 37, 31-46. Doi: https://doi.org/10.1002/(SICI)1099-050X(199821)37:1<31::AID-HRM4>3.0.CO;2-W

Cohen, W.M., Levinthal, D.A. (1990). Absorptive capacity: a new perspective of learning and innovation. Administrative Quaterly 35 (1). 128-152. Doi: https://doi.org/10.2307/2393553

Corrales, R. (2013). Creación de Capacidades y Competencias para la Innovación del Sector Turístico Costarricense y sus Implicaciones sobre el Desarrollo Local. Tesis de Posgrado, Universidad Nacional de Costa Rica. 
Revista de Política Económica • EISSN: 2215-4167

Vol. 1 (1) • Julio-Diciembre, 2015: 1-19

DOI: http://dx.doi.org/10.15359/peds.1-1.2

Ruiz Mejías y Corrales Mejías

URL: http://www.revistas.una.ac.cr/politicaeconomica

Corrales, R. (2014). Propuestas de Política Pública para Procesos de Innovación Inclusiva en el Sector Turístico Costarricense. En Análisis Público (3), Universidad Valparaíso, Chile. Recuperado de: http://analisispublico.administracionpublica-uv.cl/wp-content/ uploads/2014/06/05-III.pdf

Dijk, M.P., \& Sandee, H. (2002). Innovation and small enterprises development in developing countries. En Dijk M.P., Sandee, H. Eds. Innovation and small enterprises in the third world. Chapter 1. Ed. Edward Elgar. UK.

Dosi, G. (1988). Sources, procedures, and microeconomic effects of innovation. Journal of Economic Literature 26 (3). 1120-1171. Retrieved from: http://dimetic.dime-eu.org/ dimetic files/DosiJEL1988.pdf

Drazin, R., \& Rao, H. (2002). Harnessing managerial knowledge to implement product-line extensions: How do mutual fund families allocate portfolio managers to old and new funds? Academy of Management Journal, 45, 609-619. Doi: https://doi.org/10.2307/3069385

Edquist, C. (Ed.) (1997). Systems of Innovations: Technologies, Institutions and Organizations. Wiltshire, UK: John de la Mothe Series Editor. Retrieved from: https://charlesedquist.files. wordpress.com/2015/06/science-technology-and-the-international-political-economyseries-charles-edquist-systems-of-innovation -technologies-institutions-and-organizations-routledge-1997.pdf

Edquist, C., Hommen, L., \& McKelvey, M. (2001). Innovation and Employment: Process versus Producto Innovation. Massachusetts: Edward Elgar Publishing Limited. Doi: https://doi. org/10.4337/9781843762874

Freeman, D.A. (2006). On The So-Called: "Huber Sandwich Estimator" and "Robust Standar Error". En: The American Statician 60 (4), 299-302.

Freeman. C. (1987). Technology Policy and Economic Performance: Lessons from Japan. London: Pinter, Publisher.

Gonzáles, C. (2010). Sesgo de Selección Muestral con STATA. Colombia: Universidad ICESI, Departamento de Economía.

Grant, R. M. (1997). The knowledge-based view of the firm: Implications for management practice. Long Range Planning, 30(3), 450-454. Doi: https://doi.org/10.1016/S0024-6301(97)00025-3

Gujarati, D. (2010). Econometría. (3 ${ }^{\text {er }}$ Ed.) México: McGraw Hill.

Heckman, J. J. (1979). Sample selection bias as a specification error. En Econometrica, 47(1), 153-161. Doi: https://doi.org/10.2307/1912352 
Revista de Política Económica • EISSN: 2215-4167

Vol. 1 (1) • Julio-Diciembre, 2015: 1-19

DOI: http://dx.doi.org/10.15359/peds.1-1.2

Ruiz Mejías y Corrales Mejías

URL: http://www.revistas.una.ac.cr/politicaeconomica

Henderson, R. M., \& Clark, K. B. (1990). Architectural innovation: The reconfiguration of existing product technologies and the failure of established firms. Administrative Science Quarterly, 35, 9-30. Doi: https://doi.org/10.2307/2393549

Hitt, M. A., Bierman, L., Shimizu, K., \& Kochhar, R. (2001). Direct and moderating effects of human capital on strategy and performance in professional service firms: A resourcebased perspective. Academy of Management Journal, 44(1),13-28. Doi: https:/doi. org/10.2307/3069334

Knudsen, H. (1995). Employee Participation in Europe. London: SAGE Publications.

Kogut, B., \& Zander, U. (1992). Knowledge of the firm, combinative capabilities, and the replication of technology. Organization Science, 3, 383-397. Doi: https://doi.org/10.1287/ orsc.3.3.383

Lam, A. (2002). Alternative Societal Models of Learning and Innovation in the Knowledge Economy. Denmark: DRUID, Working Paper. Retrieved from: http://onlinelibrary.wiley. com/doi/10.1111/1468-2451.00360/pdf

Lam, A. (2010). Innovative Organizations: Structure, Learning and Adaptation. En: Innovation Perspectives for the 21st Century. Madrid: BBVA, Spain, 163-175. Retrieved from: https:// pdfs.semanticscholar.org/40f9/6a8deddd30315b40d4dce9fd01ba0ba170c1.pdf

Lazear, E. (1999). Globalizations and the market for team-mates. Economic Journal 109, 15-40. Doi: https://doi.org/10.1111/1468-0297.00414

Lundvall, B. (2007). Higher Education, Innovation and Economic Development. Aalborg University, Denmark. Working Paper.

Lundvall, B.-Å. (Ed.) (1992). National Systems of Innovation: Towards a Theory of Innovation and Interactive Learning. London: Pinter Publishers. Doi: https://doi.org/10.1016/0048$\underline{7333(95) 90017-9}$

Lundvall, B.-Å. (Ed.) (2002). Growth Innovation and Social Cohesion: The Danish Model. Elgar Publisher. Doi: https://doi.org/10.4337/9781781008348

Lundvall, B.-Å., Johnson, B., Andersen, E.S., \& Dalum, B. (2001). National System of Production, Innovation and Competence Building. Denmark: Department of Business Studies, Aalborg University. Retrieved from: http://www.obs.ee/ siim/seminars/lundvall\%2B2002.pdf

Ministerio de Ciencia, Tecnología y Telecomunicaciones [MICITT] (2014). Indicadores Nacionales de Ciencia, Tecnología e Innovación 2012-2013. Ministerio de Ciencia, Tecnología y Telecomunicaciones, Costa Rica. 
Revista de Política Económica • EISSN: 2215-4167

Vol. 1 (1) • Julio-Diciembre, 2015: 1-19

DOI: http://dx.doi.org/10.15359/peds.1-1.2

Ruiz Mejías y Corrales Mejías

URL: http://www.revistas.una.ac.cr/politicaeconomica

Nelson, R. (1993). National Innovation Systems: A Comparative Analysis. Illinois: Columbia University.

Nonaka, I. (1991). A dynamic theory of knowledge creation. En Organization Science (5) 14-37. Retrieved from: https://www.jstor.org/stable/pdf/2635068. pdf?refreqid=excelsior\%3A169c8594fbc15febdfdea4a9760ce331

Nonaka, I., \& Takeuchi, H. (1995). The knowledge-creating company. New York: Oxford University Press.

Orozco, J. (2002). Innovation and performance improvements in the Co-operative sector: Costa Rica. Tesis de grado para optar por título de Ph.D. Aalborg, University, Denmark.

Østergaard, C.R., Timmermans, B., \& Kristinsson, K. (2011). Does a different view create something new? The effect of employee diversity on innovation. Research Policy 40, 500509. Doi: https://doi.org/10.1016/j.respol.2010.11.004

Parrotta, P., Pozzoli, D., \& Pytlikova, M. (2014). The nexus between labor diversity and firm's innovation. J Popul Econ 27, 303-364. Doi: https://doi.org/10.1007/s00148-013-0491-7

Quintana-Garca, C. y Benavides-Velasco, C.A. (2008). Innovative competence, exploration and exploitation: the influence of technological diversification. Research Policy 37, 492-507. Doi: https://doi.org/10.1016/j.respol.2007.12.002

Ruef, M., Aldrich, H., \& Carter, N. (2003). The estructure of founding teams: Homophily strong ties and isolation among U.S. entrepreneurs. American Sociological Review 68 (2), 195-222. Doi: https://doi.org/10.2307/1519766

Ruiz, K. (2007). Costa Rica as a Learning Economy: An Exploratory Study of CompetenceBuilding and the Significance of Labour Relations and Labour Market Institutions. PhD. Thesis. Aalborg University, Denmark.

Schumpeter, J.A. (1934). The Theory of Economic Development: An Inquiry into Profits, Capital, Credit, Interest, and Business Cycle. Cambridge, MA: Harvard University Press.

van der Vegt, G., \& Janssen, O. (2003). Joint impact of interdependence and group diversity on innovation. Journal of Management 29 (5), 729-751. Doi: https://doi.org/10.1016/S0149-2063 03 00033-3

Wenger, E. (2000). Communities of practise and social learning systems. Organization 7 (2), 225-246. Doi: https://doi.org/10.1177/135050840072002

Wiig, K. M. (1997). Integrating intellectual capital and knowl- edge management. Long Range Planning, 30, 399-405. Doi: https://doi.org/10.1016/S0024-6301(97)90256-9 https://doi.org/10.1016/S0024-6301(97)00020-4 
Revista de Política Económica • EISSN: 2215-4167

Vol. 1 (1) • Julio-Diciembre, 2015: 1-19

DOI: http://dx.doi.org/10.15359/peds.1-1.2

Ruiz Mejías y Corrales Mejías

URL: http://www.revistas.una.ac.cr/politicaeconomica

Woodman, R., Sawyer, J., \& Griffin, R. (1993). Toward a theory of organizational creativity. The Academy of Management Review 18 (2), 293-321. Doi: https://doi.org/10.2307/258761 https://doi.org/10.5465/AMR.1993.3997517

Wooldridge, J. (2006). Introducción a la Econometría: Un enfoque moderno. México: Ed. Thompson Learning. Recuperado de: http://aulavirtual.usac.edu.gt/cursoseco/pluginfile. $\mathrm{php} / 2279 / \mathrm{mod}$ resource/content $/ 0 /$ INTRODUCCI\%C3\% 93N\%20A\%20LA\%20 ECONOMETR\%C3\%8DA\%20\%E2\%80\%93\%20UN\%20ENFOQUE\%20MODERNO.pdf

Zúñiga Vargas, F. (2004). Why Labour Competence? Key Elements to be considered. Montevideo: ILO/CINTEFOR. 